\title{
Dimorfismo sexual em Hepatus pudibundus (Crustacea, Decapoda, Brachyura)
}

\author{
Murilo Z. Marochi' ', André Trevisan², Felipe B. Gomes \& Setuko Masunari
}

\author{
1. Programa de Pós-Graduação em Zoologia, Universidade Federal do Paraná, Av. Cel. Francisco H. dos Santos, 210, Caixa Postal 19020, $81531-970$, Curitiba, PR \\ Brasil. (murilomz2@hotmail.com; set_mas@ufpr.br) \\ 2. Departamento de Ciências Biológicas, Universidade do Alto Vale do Rio do Peixe, Rua Victor Baptista Adami, 800, 89500-000, Caçador, SC, Brasi \\ (trevisan_andre@yahoo.com.br) \\ 3.Programa de Iniciação Cientííca, Universidade Federal do Paraná. (felipeb.gomes92@gmail.com)
}

Recebido 14 janeiro 2015

Aceito 15 abril 2016

DOI: $10.1590 / 1678-4766 e 2016003$

\begin{abstract}
Sexual dimorphism in Hepatus pudibundus (Crustacea, Decapoda: Brachyura). A study on sexual dimorphism in Hepatus pudibundus (Herbst, 1785) was performed using geometric morphometrics. The carapace of 28 males and 30 females and the left and right cheliped propodus of 22 males and 26 females were analyzed. Thirteen landmarks were established in the carapace and 10 in the cheliped propodus. A Generalized Procrustes Analysis based on landmark configurations was used to separate the components of size and shape. A Student t-test was used to determine the statistically significant sexual dimorphism of the carapace and the cheliped propodus. The variation in the shape of the structures was evaluated with a discriminant analysis. There was sexual dimorphism in the analyzed structures. Males were smaller than females. The carapace of females was larger in the posterior region than in males, indicating an expansion of the area for accommodation of the egg mass. The fixed finger of the cheliped propodus of females is more posteriorly arched that can be used to facilitate the cleaning of eggs laid in the abdominal chamber. Our results provide new information about the development of sexual secondary characters and their effects on the shape of the carapace and the cheliped propodus in males and females of $H$. pudibundus.
\end{abstract}

KEYWORDS. Aethridae, carapace, propodus, geometric morphometrics, morphological variation.

RESUMO. Um estudo sobre o dimorfismo sexual no caranguejo Hepatus pudibundus (Herbst, 1785) foi realizado utilizando morfometria geométrica. A carapaça de 28 machos e 30 fêmeas e o própodo dos quelípodos direito e esquerdo de 22 machos e 26 fêmeas foram analisados. Foram estabelecidos 13 marcos anatômicos bidimensionais na carapaça e 10 no própodo para ambos os sexos. Uma Análise Generalizada de Procrustes baseada na configuração dos marcos anatômicos foi utilizada para separar os componentes de tamanho e de forma. Um Teste-t de Student foi utilizada para determinar a significância estatística do dimorfismo sexual da carapaça e do própodo dos quelípodos. A variação na forma das estruturas foi avaliada através de uma Análise de Função Discriminante. Houve dimorfismo sexual na forma e no tamanho das estruturas analisadas. Machos mostraram dimensões menores do que fêmeas. A carapaça das fêmeas foi mais larga na região posterior do que em machos, indicando uma ampliação da área para acomodação da massa de ovos. O dedo fixo do própodo dos quelípodos das fêmeas está mais voltado para a parte posterior e pode facilitar a limpeza dos ovos fixados na câmara abdominal. Nossos resultados trazem novas informações sobre o desenvolvimento de caracteres sexuais secundários e as suas consequências sobre a forma da carapaça e o própodo dos quelípodos em machos e fêmeas de $H$. pudibundus.

PALAVRAS-CHAVE. Aethridae, carapaça, própodo do quelípodo, morfometria geométrica, variação morfológica.

A dimorfismo sexual pode ser definido como diferenças morfológicas entre os sexos que estão relacionados principalmente à seleção sexual, favorecendo distintas formas e tamanhos corpóreos. Em Decapoda Brachyura, esta característica é amplamente conhecida pelas variações na forma e no tamanho do abdômen e do própodo dos quelípodos, como nos caranguejos do gênero $U c a$ (Leach, 1814) cujo macho que pode apresentar o quelípodo maior, até 30 vezes maior (comprimento e altura) que o da fêmea (MASUnARi \& Swiech-Ayoub, 2003).
Técnicas de morfometria geométrica são utilizadas para verificar diferenças significativas na forma e no tamanho de determinadas estruturas corpóreas, para responder questões relacionadas às variações intra e interespecíficas, questões evolutivas e ecológicas de maneira geral (KLINGENBERG, 2010; Mitroviski-Bogdanović et al., 2014; Bower \& PILler, 2015), como a alometria ontogenética e o dimorfismo sexual (Trevisan et al., 2012; Ponssa \& CANDioti, 2012; Accioly et al., 2013). Essa técnica tem incrementado o poder de discriminação entre os sexos, pela identificação de 
diferenças morfológicas mínimas em estruturas corporais, e tem sido utilizada em Decapoda principalmente devido ao rígido exoesqueleto e à facilidade na obtenção de marcos anatômicos (RoHLF \& MARCus, 1993; AlencAR et al., 2014). Com a compreensão da variação morfológica de caracteres homólogos é possível discutir o direcionamento da seleção sexual com base na morfologia de cada sexo bem como inferir sobre as pressões ecológicas (TATSUTA et al., 2004).

Aethridae atualmente é composta por 37 espécies. Hepatus pudibundus (Herbst, 1785) pode ser encontrado em fundos lamosos e arenosos em águas rasas, com distribuição no Atlântico Ocidental, da Géorgia (Estados Unidos) até o Rio Grande do Sul (Brasil). A espécie tem importância na composição da fauna acompanhante na pesca de arrasto do camarão Xiphopenaeus kroyeri (Heller, 1862), porém não apresenta interesse comercial direto. Os trabalhos existentes envolvendo $H$. pudibundus trazem informações sobre distribuição, estrutura populacional, reprodução, desenvolvimento, crescimento e mortalidade, porém, não existem informações sobre o dimorfismo sexual utilizando a morfometria geométrica (MANTELATTO et al., 1995a,b; Melo, 1996; Reigada \& Negreiros-Fransozo, 2000; GraÇALOPES et al., 2002; HEBLING \& RIEGER, 2003; FrACASSO \& Branco, 2005; NG et al., 2008; KLOH \& Di BeneditTo, 2010; Lima et al., 2014).

O objetivo deste estudo consiste em verificar o dimorfismo sexual na forma e no tamanho da carapaça e do própodo dos quelípodos em uma população de $H$. pudibundus, através da morfometria geométrica, testando a hipótese de que diferenças morfológicas entre os sexos tem um importante papel na seleção sexual para a espécie, como ocorre em insetos (TATSUTA et al., 2004; SANAEI et al., 2015).

\section{MATERIAL E MÉTODOS}

Amostragem. Foram obtidos 58 caranguejos adultos (28 machos e 30 fêmeas) em águas costeiras ao largo do município de Pontal do Sul, Paraná, Brasil (25³6'16"S, $48^{\circ} 23^{\prime} 20^{\prime}$ 'O), por meio de uma rede de arrasto (23 $\mathrm{mm}$ de abertura de malha) acoplada a um barco em 4 de maio de 2010. Em laboratório, os caranguejos foram identificados com base em Melo (1996) e preservados em álcool 70\%. A preservação dos caranguejos em etanol antes da analise dos dados pode alterar o tamanho dos animais, mas essa alteração é pequena, como observado por RUFINo et al. (2004) com o siri Liocarcinus depurator (Linnaeus, 1758), que apresentou um encolhimento do tamanho de cerca de $0,20 \%$ comparando o tamanho antes da preservação em etanol $70 \%$. De qualquer maneira, mesmo que esse pequeno encolhimento afete a forma, esta deve afetar igualmente machos e fêmeas do presente estudo.

Somente animais adultos e em perfeito estado de conservação (indivíduos com apêndices em regeneração foram excluídos das analises) de ambos os sexos foram considerados no presente estudo. Foram considerados somente indivíduos adultos de ambos os sexos, com largura da carapaça maior que $4 \mathrm{~cm}$ (FracAsso \& Branco, 2005; Kloh \& Di BeneditTo, 2010).
Morfometria geométrica. Imagens da carapaça (28 machos e 30 fêmeas) e dos quelípodos (22 machos e 26 fêmeas) de $H$. pudibundus foram obtidas em vista dorsal com uma câmera digital com resolução de 10 megapixels.
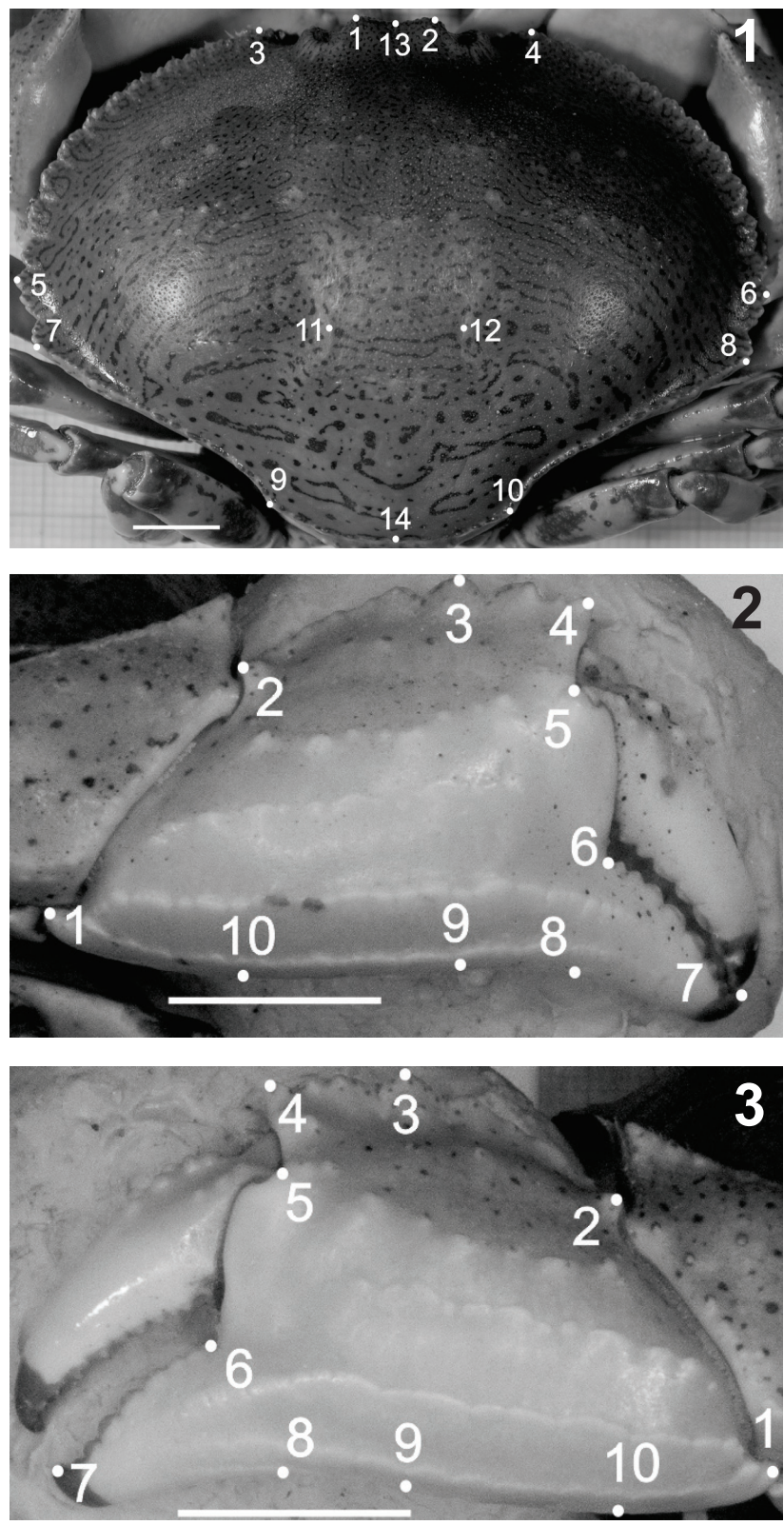

Figs 1-3. Hepatus pudibundus (Herbst, 1785), macho: Fig. 1, posição dos marcos anatômicos da carapaça [1 e 2, margem da base proximal dos lóbulos interorbitais; 3 e 4, primeiro dente anterolateral; 5 e 6 , maior largura da carapaça $\left(12^{\circ}\right.$ dente anterolateral); 7 e 8 , último dente anterolateral; 9 e 10, tubérculos da região posterior da carapaça; 11 e 12, cruzamento de sulcos hepáticos (região hepática); 13, depressão interorbital; 14, centro da margem posterior da carapaça. Fig. 2, posição dos marcos anatômicos do própodo do quelípodo direito [1, extremidade do dente distal inferior do própodo; 2 , extremidade do dente distal superior; 3 , extremidade do maior dente $\left(3^{\circ}\right)$ da crista do própodo; 4 , extremidade do primeiro dente da crista do própodo; 5 , sulco na base da margem do própodo próximo ao dedo móvel; 6 , sulco na base do primeiro dente do dedo fixo do própodo; 7 , extremidade do dedo fixo; 8 , extremidade angular entre o dedo fixo e a palma do própodo; 9 , linha vertical em relação ao maior dente da crista do própodo; 10, angulação máxima da palma do própodo]. Fig. 3, posição dos marcos anatômicos do própodo do quelípodo esquerdo (numeração dos marcos anatômicos é a mesma adotada na Fig. 2). Escalas 10 mm. 
Foram estabelecidos 13 marcos anatômicos bidimensionais na carapaça e 10 no própodo dos quelípodos direito e esquerdo (Figs 1-3).

Os marcos anatômicos foram digitalizados pelo mesmo pesquisador três vezes com a utilização do software TPS Dig2, versão 2.16 (RoHLF, 2010). Uma Análise Generalizada de Procrustes (GPA), que separa os componentes de tamanho e forma foi utilizada para realizar a sobreposição das configurações dos marcos anatômicos. A GPA consiste em sobrepor as coordenadas dos marcos anatômicos pelo centróide (centro de massa de uma configuração), proporcionar o tamanho do centróide de cada configuração para o valor de um e por último rotacionar, de modo que os marcos anatômicos correspondentes se ajustem pela menor distância quadrada possível. Desta forma é possível remover os efeitos de posição, sentido e tamanho das configurações dos marcos anatômicos, separando os componentes de tamanho e forma (MonTEIRO \& ReIS, 1999; ADAMs et al., 2004).

A carapaça é um objeto simétrico, ou seja, a estrutura em si possui um lado direito e esquerdo separado por um eixo médio, de tal modo que, os componentes de formas podem ser particionados em componentes simétricos e assimétricos (KuINGERBERG et al., 2002). Para a análise do dimorfismo da forma foram utilizados somente os componentes simétricos da carapaça. $\mathrm{O}$ tamanho de cada estrutura foi estimado através do tamanho do centróide, que é a raiz quadrada da soma das distâncias quadradas de um grupo de pontos até o seu centróide (MonTeIro \& REIS, 1999).

Análise dos dados. Para verificar diferenças no tamanho centróide da carapaça e dos quelípodos de machos e fêmeas, foi aplicado um teste t de Student. As análises foram realizadas com o auxílio do software R (R Development Core Team, 2011).

Para avaliar a ocorrência de dimorfismo sexual na forma, foi conduzida uma Análise de Componentes Principais (PCA) sobre a matriz de resíduos da GPA e os escores utilizados como novas variáveis de forma. Esse procedimento permite que os escores sejam como variáveis independentes, reduzindo a dimensionalidade dos dados. Diferenças entre as formas foram testadas com aplicação de uma Análise Discriminante (DA) com a utilização de um teste de permutação, a qual computa o percentual de classificação correta dentro de cada grupo e realiza um teste de validação cruzada entre eles (KLINGENBERG \& MonteIro, 2005). As análises foram efetuadas com o auxílio do programa MorphoJ (KLINGENBERG, 2011).

\section{RESULTADOS}

Dimorfismo sexual no tamanho. O tamanho centróide da carapaça diferiu significativamente entre os sexos, tendo as fêmeas exibido o maior valor $(\mathrm{t}=5,03$; $\mathrm{p}<$ 0,001: machos: $8,40 \pm 0,19 \mathrm{~cm}$ e fềmeas: $9,60 \pm 1,80 \mathrm{~cm}$ ). $\mathrm{O}$ tamanho do própodo dos quelípodos direito e esquerdo também diferiu significativamente entre machos e fêmeas (direito $t=5,45 ; \mathrm{p}<0,001$; esquerdo $t=4,67 ; \mathrm{p}<0,001$ ).
Fêmeas apresentaram o própodo do quelípodos esquerdo e direito maior que machos (direito: fêmeas $=3,69 \pm 1,33$ $\mathrm{cm}$ e machos $=2,64 \pm 0,99 \mathrm{~cm}$; esquerdo: fêmeas $=3,72$ $\pm 1,36 \mathrm{~cm}$ e machos $=2,77 \pm 1,02 \mathrm{~cm}$ ). Porém, os machos demonstraram própodos dos quelípodos proporcionalmente mais longos (em relação à largura da carapaça) que as fêmeas.

Dimorfismo sexual na forma. $O$ formato da carapaça diferiu significativamente entre machos e fềmeas (Distância de Procrustes $=0,020 ; p<0,01$; Distância de Mahalanobis = 3,74; $<<0,01$ ), com um percentual de classificação correta de $95,56 \%$ para as fêmeas e $95,24 \%$ para os machos. O dimorfismo sexual está principalmente relacionado à variação nos marcos anatômicos 5, 7 e 9 (e seus homólogos 6, 8 e 10, respectivamente), além do 14 (Fig. 4). As fêmeas apresentaram a margem lateral posterior menos larga que machos, cujo estreitamento provoca uma deformação na região posterior da carapaça que se torna mais longa (principalmente na região nos marcos anatômicos 9, $10 \mathrm{e}$ 14). A região anterior da carapaça apresenta deformações menos perceptíveis, tendo as fêmeas apresentado a região anterolateral da carapaça levemente mais estreita (marcos anatômicos 3 e 4). Não foram observadas diferenças na região central da carapaça (marcos anatômicos 11 e 12).

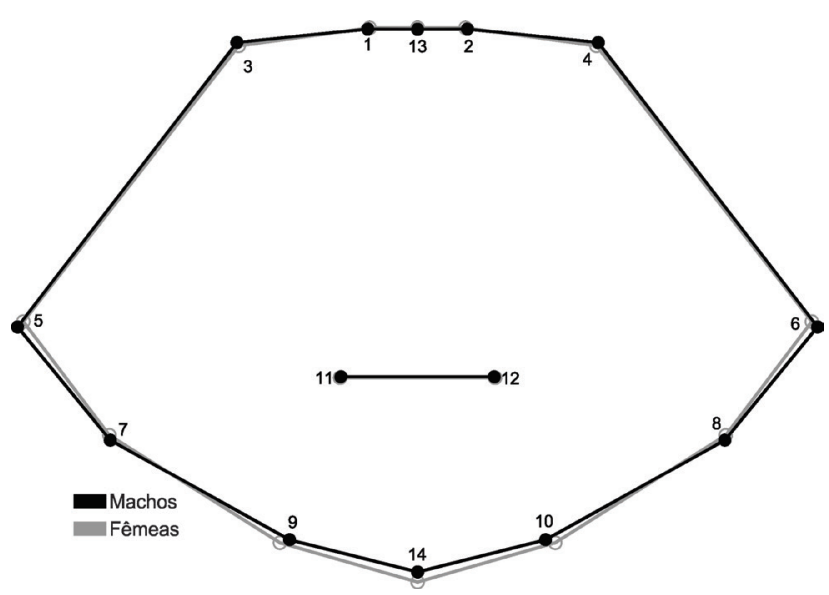

Fig. 4. Hepatus pudibundus (Herbst, 1785). Dimorfismo sexual na forma da carapaça. Deformações magnificadas uma vez.

A forma do própodo do quelípodo diferiu entre os sexos tanto para o lado direito (Distância de Procrustes = 0,$03 ; \mathrm{p}<0,01 ;$ Distância de Mahalanobis $=3,95 ; \mathrm{p}<0,01$ ) quanto para o esquerdo (Distância de Procrustes $=0,03 ; \mathrm{p}<$ 0,01 ; Distância de Mahalanobis $=4,35 ; \mathrm{p}<0,01$ ). O quelípodo direito apresentou percentual de classificação correta de $100 \%$ para fêmeas e $90,91 \%$ para machos, enquanto para o quelípodo esquerdo, esses valores foram de 96,30 e 93,94\%, respectivamente para fêmeas e machos.

Os própodos de ambos quelípodos apresentaram o mesmo padrão de variação. Fêmeas apresentaram a margem interna do quelípodo mais curvada na região dos marcos anatômicos 8 e 9, enquanto os machos, em forma mais linear. Essa curvatura está associada à deformação observada no 


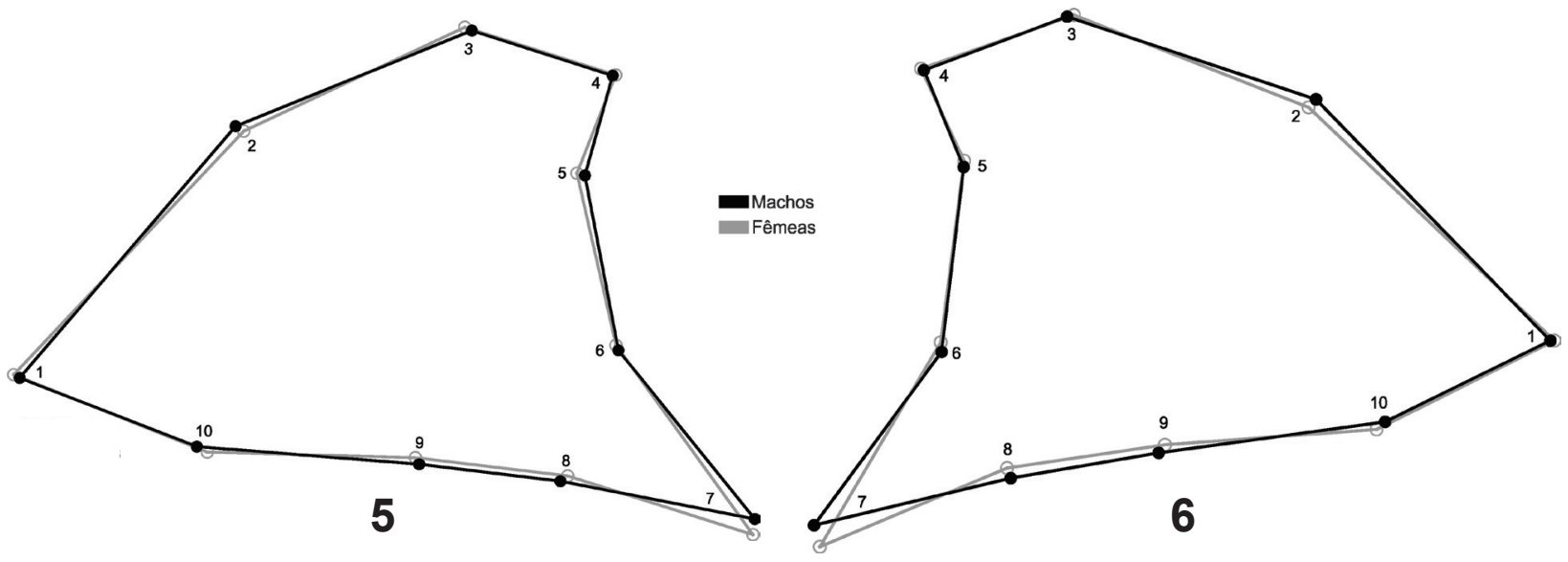

Figs 5, 6. Hepatus pudibundus (Herbst, 1785). Dimorfismo sexual na forma: 5, própodo direito; 6, própodo esquerdo. Deformações magnificadas uma vez.

dedo fixo do quelípodo no marco anatômico 7. Por outro lado, machos apresentam a margem externa do própodo do quelípodo levemente mais curvada (marco anatômico 2) do que as fêmeas (Figs 5, 6).

\section{DISCUSSÃO}

Nós encontramos dimorfismo sexual no tamanho e forma da carapaça e do própodo dos quelípodos em Hepatus pudibundus. Esta variação está relacionada às distintas funções e adaptações reprodutivas dos sexos e pode ser uma consequência das diferentes estratégias sexuais do grupo, uma vez que a energia para o crescimento é usada de forma distinta em machos e fêmeas. Os comportamentos de forrageamento, reprodutivos e agonísticos são considerados as principais pressões seletivas que direcionam respostas evolutivas para ambos os sexos em Brachyura (LEE, 1995; Alencar et al., 2014). O dimorfismo sexual pode ser uma consequência direta de hormônios específicos a cada sexo, que proporcionam uma morfologia distinta relacionada ao sucesso reprodutivo da espécie.

A variação na forma da carapaça e dos própodos entre os sexos ocorre como uma consequência do crescimento relativo diferenciado das dimensões corpóreas durante a ontogênese, e esta pode ser regulada pela presença ou ausência de hormônios andrógenos (HARTNOLL, 1974, 1978; SAGI et al., 1997). A forma mais ampla da região posterior da carapaça nas fêmeas está relacionada ao aumento do potencial reprodutivo da espécie, ampliando a área para acomodação e proteção, bem como facilitando a fixação da massa de ovos no abdômen, corroborando o padrão para espécie (HARTNOLL, 1974; Lima et al., 2014). Em trabalhos sobre dimorfismo sexual utilizando morfometria geométrica, o mesmo padrão de variação morfológica para fêmeas foi observado para Liocarcinus depurator (Linnaeus, 1758) (RuFINo et al., 2004a,b, 2006) e Ucides cordatus (Linnaeus, 1763) (AlENCAR et al., 2014).
Embora em valores absolutos o tamanho do própodo dos quelípodos tenha se apresentado maior nas fêmeas, ele foi proporcionalmente maior (em relação ao tamanho da carapaça) dentre os machos: um padrão esperado para os Brachyura e confirmado para a espécie (LIMA et al., 2014). Este tamanho proporcionalmente maior pode representar vantagens durante interações intraespecíficas como comportamento agonístico, defesa contra predadores e processos relacionados à reprodução (LiMA et al., 2014).

A forma dos quelípodos direito e esquerdo foi similar em machos e fêmeas, mas distintos entre os sexos, como esperado para uma espécie que não apresenta heteroquelia. O dedo fixo do própodo dos quelípodos voltado para a parte posterior dentre as fêmeas pode ser utilizado como um facilitador em alcançar a câmara abdominal e as estruturas sensoriais no processo de limpeza dos ovos (Almerão et al., 2010). Porém, estudos futuros sobre o comportamento da espécie são necessários para confirmar esta hipótese.

O tamanho maior das fêmeas em relação aos machos não seguiu o padrão para a espécie quando comparados com o tamanho médio da largura da carapaça entre os sexos, para populações do Rio de Janeiro, São Paulo (Mantelatto et al., 1995a; KeUnecke et al., 2007; KLOH \& Di BeneditTo, 2010) e Santa Catarina (FracAsSo \& Branco, 2005). Apesar da diferença significativa de tamanho entre machos e fêmeas, ambos atingem a maturidade sexual morfológica com aproximadamente $32,5 \mathrm{~mm}$ de largura da carapaça (Reigada \& Negreiros-Fransozo, 1999). O tamanho corpóreo maior de fêmeas pode ser explicado, em parte, por fatores relacionados à variação populacional (status nutricional e disponibilidade de alimento) ou a metodologia de coleta empregada, que pode ter selecionado fêmeas maiores que machos (ANASTASIADOU et al., 2009).

De modo geral nossos resultados trazem novas informações sobre o desenvolvimento de caracteres secundários e as suas consequências sobre a forma da carapaça e o própodo dos quelípodos em machos e fêmeas 
de H. pudibundus. Essas consequências refletem a tendência sexual evolutiva para a reprodução. Além disso, a técnica de morfometria geométrica provou ser eficiente para reconhecer o dismorfismo sexual na carapaça e no própodo em $H$. pudibundus com refinado detalhamento.

Agradecimentos. Ao Prof. Dr. Henry Louis Spach do Centro de Estudos do Mar da Universidade Federal do Paraná pela cessão dos espécimes utilizados. Ao Programa de Pós-graduação em Zoologia, UFPR pela utilização das instalações. Esta pesquisa foi apoiada pelo Conselho Nacional de Desenvolvimento Científico e Tecnológico (CNPq), Proc. 141212/2013-6 (primeiro autor) e 145705/2013-7 (terceiro autor). Todo material biológico coletado tem a permissão do Instituto Brasileiro do Meio Ambiente e dos Recursos Renováveis (IBAMA) (Autorização n 234821). Esta é a contribuição n 1918 do Departamento de Zoologia da UFPR.

\section{REFERÊNCIAS BIBLIOGRÁFICAS}

Accioly, I. V.; Lima-Filho, P. A.; Santos, T. L.; Barbosa, A. C. A.; Campos, L. B. S.; Souza, J. V.; Araújo W. C. \& Wagner F. M. 2013. Sexual dimorphism in Litopenaeus vannamei (Decapoda) identified by geometric morphometrics. Pan-American Journal of Aquatic Sciences 8(4):276-281.

Adams, D. C.; RohlF, F. J. \& Slice, D. E. 2004. Geometric morphometrics: ten years of progress following the 'revolution'. Italian Journal of Zoology 71:5-16.

Alencar, C. E. R. D.; Lima-Filho, P. A.; Molina, W. F. \& Freire, F. A. M. 2014. Sexual Shape Dimorphism of the Mangrove Crab Ucides cordatus (Linnaeus, 1763) (Decapoda, Ucididae) Accessed through Geometric Morphometric. The Scientific World Journal 1:1-8.

Almerão, M.; Bond-Buckup, G. \& MendonçA JR. M. DE S. 2010. Mating behavior of Aegla platensis (Crustacea, Anomura, Aeglidae) under laboratory conditions. Journal of Ethology 28(1):87-94.

Anastasiadou, C.; Liasko, R. \& Leonardos, I. D. 2009. Biometric analysis of lacustrine and riverine populations of Palemonetes antennarius $(\mathrm{H}$ Milne-Edwards, 1837) (Crustacea, Decapoda, Palaemonidae) from north-western Greece. Limnologica 39:244-254.

Bower, L. M. \& PiLLER, K. R. 2015. Shaping up: a geometric morphometric approach to assemblage ecomorphology. Journal of Fish Biology 87(3):691-714

Fracasso, H. A. A. \& Branco, J. O. 2005. Estrutura populacional de Hepatus pudibundus (Herbst) (Crustacea, Decapoda) na Armação do Itapocoroy, Penha, Santa Catarina, Brasil. Revista Brasileira de Zoologia 22(2):342-348

Graça-Lopes, R.; Tomás, A. R. G.; Tutui, S. L. dos S.; Severino Rodrigues, E. \& Puzzi, A. 2002. Fauna acompanhante da pesca camaroeira no litoral do estado de São Paulo, Brasil. Boletim do Instituto de Pesca 28(2):173-188.

HARTNOLL, R. G. 1974. Variation in growth patterns between some secondary sexual characters in crabs (Decapoda, Brachyura). Crustaceana 27:131136.

1978. The determination of relative growth in Crustacea. Crustaceana 34:281-293.

Hebling, N. J. \& Rieger, P. J. 2003. Desenvolvimento juvenil de Hepatus pudibundus (Herbst) (Crustacea, Decapoda, Calappidae), em laboratório. Revista Brasileira de Zoologia 20(3):531-539.

KeUNECKE, K. A.; D'InCAO, F. \& FonseCA, D. B. 2007. Growth and mortality of Hepatus pudibundus (Crustacea: Calappidae) in south-western Brazil. Journal of the Marine Biological Association of the UK 87:885-891.

KLOH, A. S. \& Di Beneditto, A. P. M. 2010. Estrutura populacional do siri-baú, Hepatus pudibundus (Herbst 1785) no norte do Estado do Rio de Janeiro, Sudeste do Brasil. Biota Neotropica 10(3):463-467.

KLINGENBERG, C. P. 2010. Evolution and development of shape: integrating quantitative approaches. Nature Reviews Genetics 11(9):623-635.

2011. Morpho J: an integrated software package for geometric morphometrics. Molecular Ecology Resources 11:353-357.

KlingenberG, C. P.; Barbeluga, M. \& Meyer, A. 2002. Shape analysis of symmetric structures: quantifying variation among individuals and asymmetry. Evolution 56:1909-1920.
KLingenberg, C. P. \& Monteiro, L. R. 2005. Distances and directions in multidimensional shape spaces: implications for morphometric applications. Systematic Biology 54:678-688.

LEE, S. Y. 1995. Cheliped size and structure: the evolution of a multifunctional decapod organ. Journal of Experimental Marine Biology and Ecology 193:161-173.

Lima, P. A.; Bertini, G.; Fransozo, V.; Gregati, R. A.; Corrêa FernandesGóes, L. \& CAstilho, A. L. 2014. Reproductive biology of Hepatus pudibundus (Crustacea: Brachyura), the most abundant crab on the southeastern Brazilian coast. Biologia 69(2):219-227.

Mantelatto, F. L. M.; Fransozo, A. \& Negreiros-Fransozo, M. L. 1995a. Distribuição do caranguejo Hepatus pudibundus (Herbst, 1785) (Crustacea, Decapoda, Brachyura) na Enseada da Fortaleza, Ubatuba (SP), Brasil. Boletim do Instituto Oceanográfico 43(1):59-69.

1995b. Population structure of Hepatus pudibundus (Decapoda: Calappidae) in Fortaleza Bay, Brazil. Revista de Biologia Tropical 43(1-3):265-270.

Masunari, S. \& Swiech-Ayoub, B. P. 2003. Crescimento relativo em Uca leptodactyla Rathbun (Crustacea, Decapoda, Ocypodidae). Revista Brasileira de Zoologia 20(3):487-491.

Melo, G. A. S. 1996. Manual de identificação dos Brachyura (Caranguejos e Siris) do litoral brasileiro. São Paulo, Plêiade, FAPESP. 604p.

Mitrovski-Bogdanović, A.; Tomanović, Ž.; Mitrović, M.; Petrović, A.; IVAnović, A.; Žıirić, V.; Starý, P. \& Vorburger, C. 2014. The Praon dorsale-yomenae s.str. complex (Hymenoptera, Braconidae, Aphidiinae): Species discrimination using geometric morphometrics and molecular markers with description of a new species. Zoologischer Anzeiger 253(4):270-282.

Monteiro, L. R. \& Reis, S. F. 1999. Princípios de morfometria geométrica. Ribeirão Preto, Holos. 189p

NG, P. K. L.; Guinot, D. \& DaVIE, P. J. F. 2008. Systema Brachyurorum: Part 1. An Annotated checklist of extant Brachyuran crabs of the world. Raffles Bulletin of Zoology (Supplement Series) 17:1-286.

Ponssa, M. L. \& CANDioti, M. F. V. 2012. Patterns of skull development in anurans: size and shape relationship during postmetamorphic cranial ontogeny in five species of the Leptodactylus fuscus Group (Anura: Leptodactylidae). Zoomorphology 131(4):349-362.

R Development Core Team. 2011. R: A language and environment for statistical computing. Disponível em <http://www. R-project.org/>. Acesso em 13.01.2015.

Reigada, A. L. D. \& Negreiros-Fransozo, M. L. 1999. Maturidade sexual em Hepatus pudibundus (Decapoda, Brachyura, Calappidae). Iheringia, Série Zoologia (86):159-164.

2000. Reproductive cycle of Hepatus pudibundus (Herbst, 1785) (Crustacea, Decapoda, Calappidae) in Ubatuba, SP, Brazil. Revista Brasileira de Biologia 60(3):483-491.

Rohlf, F. J. 2010. TpsDig, version 2.16: A program for digitizing landmarks and outlines for geometric. Disponível em $<\mathrm{http}: / /$ life. bio.sunysb.edu/morph/index .html >. Acesso em 13.01.2015.

RoHLF, F. J. \& MARCUS, L. F. 1993. A revolution in morphometrics. Trends in Ecology and Evolution 8:129-132.

Rufino, M.; Abello, P. \& Yule, A. B. 2004a. Male and female carapace shape differences in Liocarcinus depurator (Decapoda, Brachyura): an application of geometric morphometric analysis to crustaceans. Italian Journal of Zoology 71(1):79-83.

$2004 \mathrm{~b}$. The effect of alcohol and freezing preservation on carapace size and shape in Liocarcinus depurator (Crustacea, Brachyura). In: ElewA, A. M. T. ed. Morphometrics - Applications in Biology and Paleontology. Berlin, Heidelberg Springer, p. 45-53.

2006. Geographic and gender shape differences in the carapace of Liocarcinus depurator (Brachyura: Portunidae) using geometric morphometrics and the influence of a digitizing method. Journal of Zoology 269:458-465.

SaGi, A.; SniR, E. \& Khalaila, I. 1997. Sexual differentiation in decapod crustaceans: role of the androgenic gland. Invertebrate Reproduction and Development 31(1-3):55-61.

Sanaei, E.; Seiedy, M. \& Momtazi, F. 2015. Evolutionary view on sexual dimorphism and shape variation in Iranian populations of Hypera postica (Coleoptera: Curculionidae). Zoomorphology 134(4):541-552. 
Tatsuta, H.; Mizota, K. \& Акімото, S. I. 2004. Relationship between size and shape in the sexually dimorphic beetle Prosopocoilus inclinatus (Coleoptera: Lucanidae). Biological Journal of the Linnean Society 81:219-233.
Trevisan, A.; Marochi, M. Z.; Costa, M.; Santos, S. \& Masunari, S. 2012. Sexual dimorphism in Aegla marginata (Decapoda: Anomura). Nauplius 20:75-86. 